\title{
SIGNAL PROCESSING PROBLEMS AND ALGORITHMS IN DISPLAY SIDE OF 3DTV
}

\author{
E. Ulusoy ${ }^{1}$, G.B. Esmer ${ }^{1}$, H.M. Ozaktas ${ }^{1}$, L. Onural ${ }^{1}$, A. Gotchev ${ }^{2}$ and V. Uzunov ${ }^{2}$ \\ ${ }^{1}$ Bilkent University, Department of EEE TR-06800 Bilkent, Ankara, Turkey \\ ${ }^{2}$ Tampere University of Technology, Institute of Signal Processing FIN-33101 Tampere, Finland
}

\begin{abstract}
Two important signal processing problems in the display side of a holographic 3DTV are the computation of the diffraction field of a 3D object from its abstract representation, and determination of the best display configuration to synthesize some intended light distribution. To solve the former problem, we worked on the computation of $1 \mathrm{D}$ diffraction patterns from discrete data distributed over $2 \mathrm{D}$ space. The problem is solved using matrix pseudo-inversion which dominates the computational complexity. Then, the light field synthesis problem by a deflectable mirror array device (DMAD) is posed as a constrained linear optimization problem. The formulation makes direct application of common optimization algorithms quite easy. The simulations indicate that developed methods are promising.
\end{abstract}

Index Terms - 3DTV, scalar diffraction, light field synthesis, deflectable mirror array device

\section{INTRODUCTION}

The holographic display and the associated signal processing algorithms are the integral components of a holographic 3D television system. The main function of a holographic display is to synthesize a complex light field which pretty much resembles the original light field that would emerge from a 3D scene if it was illuminated by some coherent light source, so that the observers would see the original scene without its actual presence. We firstly need some information about the diffracted light field. One practice is to extract the three dimensional information about the object itself through computer graphics techniques, and provide the display end with some abstract information such as a mesh structure with texture information, etc. Therefore, one of the main tasks of the display end becomes the computation of the diffraction field that would emanate from the $3 \mathrm{D}$ scene which is described in abstract terms. Unfortunately, computation of diffraction field over 3D space brings too much computational burden. Hence, we concentrate on the problem of calculation of $1 \mathrm{D}$ diffraction pattern on a line from distributed samples over 2D space. In most of the earlier works, straightforward superposition is

This work is supported by EC within FP6 under Grant 511568 with acronym 3DTV. used to compute of the diffraction pattern [1]. An appropriate calculation method which provides a simultaneous solution is presented in sec. 2 .

After the determination of the diffraction field of the object, the subsequent task is to synthesize this field. Given the diffraction field, one must compute the driving signals or optimum configuration for the display such that the intended field is recreated to the best extent. We dealt with this second main signal processing problem in sec. 3 . We chose DMAD as the display device. The mirrors on this device can be tilted to control the output light field. Firstly, we analyzed the light field generated by this device upon a certain configuration. Secondly, for this device, we formulated the light field synthesis problem in a linear constrained optimization problem framework, for which a myriad of optimization algorithms exist and can be adapted.

\section{CALCULATION OF SCALAR OPTICAL DIFFRACTION DUE TO DISCRETE DATA}

Calculation of scalar optical diffraction pattern on a line due to a set of discrete data distributed over 2D space is a tricky problem. Superposition of the individual diffraction patterns of the data points may give erroneous results. To justify this fact, two scenarios will be investigated. In the first scenario, the field is completely specified on all the data points of the input line which is parallel to the output line. In this scenario, the problem can be formulated within a linear shiftinvariant system framework, so the diffraction pattern on the output line can be calculated by convolving the input pattern and the diffraction kernel. In the second scenario, the field is not specified on one of the sample locations of the input line. Instead, an additional value is provided at a sample location outside the input line, to compensate the information loss due to the missing data. In this scenario, the data on the input line and the complementary data are affected by each other. Hence, the method that calculates the diffraction field should take this interaction into account. Here, we propose such a method. Given discrete data is arbitrarily distributed over $2 \mathrm{D}$ space and we use parallel lines to represent the $2 \mathrm{D}$ space. Some of the samples on each parallel line are specified while others are unknown. Figure 1 illustrates a typical case. As it can be seen from Figure 1, each line contains black 


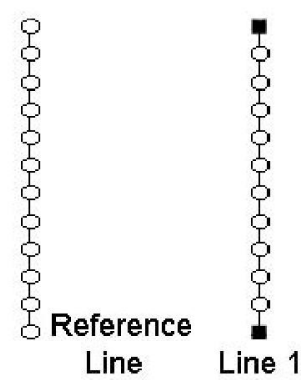

Line

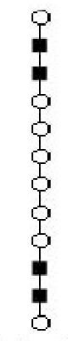

Line 2

Fig. 1. An Illustration of a set of distributed data over 2D space.

squares and circles. Black squares stand for known samples, while circles represent the missing data. Using the available data, our method calculates the samples of the diffraction field on a separate reference line.

\subsection{Basic Theory}

The relation between diffraction patterns on the input line and the output line is

$$
u(x, z)=h_{z}(x) * u(x, 0)
$$

where $u(x, 0)$ represents the diffraction pattern on the input line which is located at $z=0$ and $u(x, z)$ is the calculated diffraction pattern on the output line which is parallel to the input line [2]. Spatial domain coordinates are represented by the variables $x$ and $z$. The diffraction kernel under Fresnel approximation is

$$
h_{z}(x)=\frac{e^{j k z}}{\sqrt{j \lambda z}} e^{j \frac{k}{2 z} x^{2}}
$$

where the parameter $k$ is the wave number. The parameter $\lambda$ is the optical wavelength. As it can be seen from Eq. 1 and Eq. 2, when the input and the output lines are parallel to each other, the diffraction kernel becomes shift-invariant. Moreover, if we know the data on a line completely, we can compute the diffraction pattern over the entire $2 \mathrm{D}$ space by using the relationship given by Eq. 1 .

The proposed method is based on discrete computations. Therefore, Eq. 1 is sampled and we get,

$$
\mathrm{g}=\mathbf{B f}
$$

where B represents the sampled diffraction kernel. Details of sampling procedure is given in [3]. Vectors $\mathbf{f}$ and $g$ are the samples of $u(x, 0)$ and $u(x, z)$, respectively. The diffraction patterns on $u(x, 0)$ and $u(x, z)$ are uniformly sampled. Ma- trix $\mathbf{B}$ which provides the transformation from $\mathbf{f}$ to $\mathrm{g}$ is

$$
\mathbf{B}=K\left[\begin{array}{cccc}
e^{j \frac{\pi}{N} \zeta d_{0}} & e^{j \frac{\pi}{N} \zeta d_{1}} & \ldots & e^{j \frac{\pi}{N} \zeta d_{N-1}} \\
e^{j \frac{\pi}{N} \zeta d_{1}} & e^{j \frac{\pi}{N} \zeta d_{0}} & & e^{j \frac{\pi}{N} \zeta d_{N-2}} \\
\vdots & & \ddots & \vdots \\
e^{j \frac{\pi}{N} \zeta d_{N-1}} & e^{j \frac{\pi}{N} \zeta d_{N-2}} & \cdots & e^{j \frac{\pi}{N} \zeta d_{0}}
\end{array}\right]
$$

where the term $K$ provides constant phase shift and magnitude attenuation. The variable $d_{(m-n)}$ is equal to $(m-n)^{2}$ where $m$ and $n$ are the indices of the vectors $\mathbf{f}$ and $g$, respectively. The variable $N$ is the number of samples on a line. The parameter $\zeta=\frac{N X^{2}}{\lambda z}$ where $X$ is the spatial sampling period along $x$-axis.

We define another matrix, $B_{B F}$, from the row vectors of the $B$ matrices. $B_{B F}$ gives the relationship between the diffraction field on the reference line and the distributed data over $2 \mathrm{D}$ space. Dimensions of $\mathrm{B}_{\mathrm{BF}}$ is determined by the variable $N$ and the number of the given data, $s$. The vector $\mathbf{f}$ is computed by

$$
\mathbf{f}=\mathbf{B}_{\mathrm{BF}}{ }^{+} \mathbf{g}^{\prime}
$$

where $\mathbf{B}_{\mathbf{B F}}{ }^{+}$is the pseudo-inverse of $\mathbf{B}_{\mathbf{B F}}$ and the vector $\mathbf{g}^{\prime}$ represents the diffraction data over the distributed data points. The proposed method can be extended to $3 \mathrm{D}$ by storing each $2 \mathrm{D}$ diffraction field in a $1 \mathrm{D}$ vector, but in this case the dimensions of $\mathrm{B}$ and $\mathrm{B}_{\mathrm{BF}}$ may become unmanageable.

\subsection{Simulation Results}

The proposed method is tested by setting a controlled experiment. In this experiment, an initial field as shown in Figure 2 is defined on the reference line. The experiment is comprised of 256 parallel lines over 2D space with $N=256$ samples per line. After then, we randomly pick 256 data points. The spatial sampling period along the $x$-axis is set to $\lambda / 2$. The distance between the reference line and the closest line which contains the given data is set to $N \lambda / 4$ and the distance between each data line is $\lambda / 2$. $\mathbf{B}_{\mathbf{B F}}$ matrix is obtained according to the locations of the given data points. Then, Eq. 5 provides the diffraction field on the reference line. In this simulation, the parameter $s$, which is the number of the known terms, and the variable $N$ are equal. In most cases, $s$ and $N$ take different values. Therefore, we calculate the pseudo-inverse of $\mathbf{B}_{\mathrm{BF}}$ to obtain the least-square solution. Figure 2 provides the magnitude of the difference between the initial and the calculated diffraction fields on the reference line. The computational burden is estimated as $\mathcal{O}\left(s N^{2}-N^{3} / 3\right)$ when Householder transformation is used to calculate pseudo-inverse of $\mathbf{B}_{\mathrm{BF}}$.

\section{3D LIGHT FIELD SYNTHESIS WITH A DMAD}

In this part, we seek the implementation of a holographic display with a DMAD. The DMAD is a reflection-mode SLM, which consists of a two-dimensional array of square shaped 

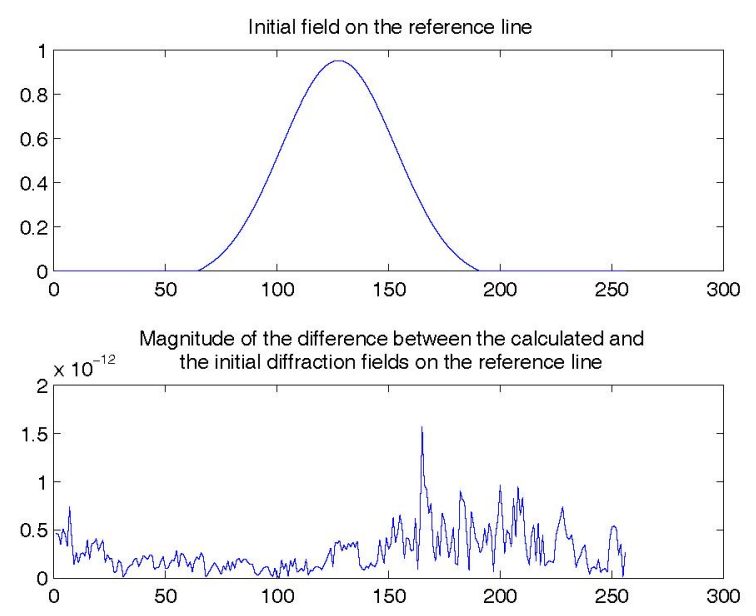

Fig. 2. Initial diffraction field on the reference line and the magnitude of the difference between the calculated and the initial diffraction fields on the reference line

micro-mirrors. The mirrors can be tilted along their diagonal axis. The deflection of each mirror can be controlled separately. Practical DMADs consist of a large number of mirrors, typically in the order of $10^{6}$ mirrors per chip. This enables them to control a large number of degrees of freedom [4].

One possible way to manipulate light with this device is by cleverly tilting the mirrors on the device and illuminating the device with laser light, so that the reflected and diffracted light will approximate an intended light field. The key issue then turns out to be the determination of the tilt angles of the mirrors according to the target field. In a 3DTV setting, this must be done within a reasonable time compatible with realtime operation. It should also be noted that the large number of mirrors on the device results in a challenging problem with very high dimensionality.

Below, we firstly provide an analysis of the field produced by this device. Secondly, we pose the light field synthesis problem as a linear constrained optimization problem.

\subsection{Light Field Generated by A DMAD}

The mirrors of the DMAD can be indexed with a column vector $\mathbf{i}=\left[\begin{array}{cc}m & n\end{array}\right]^{T}$ with $m, n \in \mathcal{Z}$, such that the i'th mirror is centered around:

$$
\mathbf{T}_{\mathbf{i}}=\left[\begin{array}{c}
x_{c} \\
y_{c} \\
0
\end{array}\right]=\left(W \sqrt{2}+\frac{L \sqrt{2}}{2}\right)\left[\begin{array}{cc}
-1 & 1 \\
1 & 1 \\
0 & 0
\end{array}\right] \mathbf{i}
$$

where $2 W$ is the width of the mirrors and $L$ is the length of the spacing between the mirrors.

After illumination with a plane wave $\exp \left\{j \frac{2 \pi}{\lambda} \kappa^{T} \mathbf{x}\right\}$, the field produced by the $i$ 'th mirror, when it is tilted by an angle $\theta_{\mathbf{i}}$ is given by:

$$
u_{\kappa}^{\left(\mathbf{i}, \theta_{\mathbf{i}}\right)}(\mathbf{x})=\exp \left\{j \frac{2 \pi}{\lambda} \kappa^{T} \mathbf{T}_{\mathbf{i}}\right\} \psi_{\mathbf{R}_{\theta_{\mathbf{i}}} \kappa}\left(\mathbf{R}_{\theta_{\mathbf{i}}}\left(\mathbf{x}-\mathbf{T}_{\mathbf{i}}\right)\right)
$$

where $\mathbf{x}$ is the position vector given by $\mathbf{x}=\left[\begin{array}{lll}x & y & z\end{array}\right]^{T}$ and $\kappa$ is the direction cosines vector of the plane wave given by $\kappa=\left[\begin{array}{lll}\alpha & \beta & \gamma\end{array}\right]^{T}$ with the constraint $\alpha^{2}+\beta^{2}+\gamma^{2}=1$. $\mathbf{T}_{\mathbf{i}}$ is the matrix given by Eq. 6 , and $\mathbf{R}_{\theta}$ is given as:

$$
\mathbf{R}_{\theta}=\left[\begin{array}{ccc}
\frac{1}{\sqrt{2}} \cos \theta & \frac{1}{\sqrt{2}} & \frac{1}{\sqrt{2}} \sin \theta \\
-\frac{1}{\sqrt{2}} \cos \theta & \frac{1}{\sqrt{2}} & -\frac{1}{\sqrt{2}} \sin \theta \\
-\sin \theta & 0 & \cos \theta
\end{array}\right]
$$

We express the results by appropriately modifying the six parameters of the function:

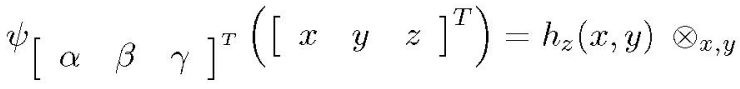

$$
\begin{aligned}
& \operatorname{rect}\left(\frac{x}{2 W}\right) \operatorname{rect}\left(\frac{y}{2 W}\right) \exp \left\{j \frac{2 \pi}{\lambda}(\alpha x+\beta y)\right\}
\end{aligned}
$$

where in our calculations $h_{z}(x, y)$ is the Fresnel impulse response of free space and $\otimes_{x, y}$ denotes $2 \mathrm{D}$ convolution in $x$ and $y$ coordinates. Detailed expressions may be found in [2].

Total field is obtained by superposing the individual contributions of mirrors.

\subsection{Linear Constrained Optimization Problem Formula- tion for Complex Light Field Synthesis with a DMAD}

Due to the quite complex and nonlinear relationship between tilt angles and produced fields (Eq. 7), direct manipulation of the expressions of previous section for the inverse problem is not a good strategy. Instead, we will consider the formulation below.

Assume the DMAD consist of $M \times N$ micromirrors and each micromirror can be tuned to $S \in \mathcal{Z}^{+}$discrete tilt angles. For convenience, let us use here a one dimensional notation for the mirrors which are finite in number. Denote the field generated by $j$ 'th mirror $(j \in \mathcal{Z}, 1 \leq j \leq M \times N)$ when it is tilted to $s^{\prime}$ th position $(s \in \mathcal{Z}, 1 \leq s \leq S)$ by $u_{j_{s}}(\mathbf{x})$, and the desired field by $b(\mathbf{x})$. Our aim is to find out the optimum tilt angle for each mirror such that $b(\mathbf{x})$ is synthesized to the best extent. There are several constraints associated with this inverse problem: during the synthesis of a frame, all the possible fields $u_{j_{s}}(\mathrm{x})$ can not be utilized simultaneously. Exactly one of the $s$ fields produced by each mirror can be employed at a time. The amplitudes of the selected fields are restricted to unity.

Upon these observations, the total field $u(\mathbf{x})$ produced by the DMAD can be expressed in the following form:

$$
u(\mathbf{x})=\sum_{j} \sum_{s} p_{j_{s}} u_{j_{s}}(\mathbf{x})
$$


Here, for each $j$, only one of the coefficients $p_{j_{s}}$ is unity, while the remaining are zero.

The next step is the digitization of the information present in the analog light fields. When Fresnel approximation is considered for diffraction, light fields emerging from spatially bounded objects can be represented fully by their samples taken at finite rates [5]. In a general case, the number of required samples is infinite, whereas, in our work, we assume that a finite number of samples are enough to characterize the fields. Thus, we represent $u_{j_{s}}(\mathbf{x})$ and $b(\mathbf{x})$ by the finite sized vectors $\mathbf{u}_{\mathbf{j}_{\mathbf{s}}}$ and $\mathbf{b}$.

After all, we get:

$$
\mathbf{D p}=\mathbf{b}
$$

where $\{(j-1) \times S+s\}$ 'th column of $\mathbf{D}$ is $\mathbf{u}_{\mathbf{j}_{\mathrm{s}}}$, and $\mathbf{p} \in$ $\mathcal{R}^{(M \times N \times S) \times 1}$ is such that for all $k \in \mathcal{Z}, 1 \leq k \leq M \times N$; the sub-vector $\mathbf{p}[(k-1) \times S+1: k \times S]$ is equal to a column of the $S \times S$ identity matrix. In words, the matrix $\mathbf{D}$ encloses the samples of different fields produced by the DMAD, hence represents the device. Vector $b$ represents the specified field. The $p$ vector, which is to be solved for, will dictate the choice of tilt positions for the mirrors. Many algorithms exist for this class of problems.

\subsection{Simulation Results}

We have carried out a simulation to illustrate the usefulness of our formulations. We considered a $71 \times 71$ DMAD with $16 \mu \mathrm{m} \times 16 \mu \mathrm{m}$ mirrors that could be tilted to $-12,0$ and 12 degrees. The interspacing between the mirrors was taken as $1 \mu \mathrm{m}$. The target field was represented by its $25 \times 25$ samples uniformly taken on a patch that resided at a distance of $z=853 \mu \mathrm{m}$ to the device. The sampling intervals became $d x=d y=53 \mu \mathrm{m}$. In particular, we tried to synthesize a circular field distribution of $1.5 \operatorname{circ}\left(\frac{\sqrt{\left(\frac{x}{d x}\right)^{2}+\left(\frac{y}{d y}\right)^{2}}}{2}\right)$. The device was normally illuminated by a plane wave of $\lambda=$ $633 \mathrm{~nm}$. We applied simulated annealing algorithm to configure the device such that the samples of the target field were reconstructed with minimum mean squared error.

To arrive at the format suggested by Eq. 11, we firstly prepared matrix $\mathbf{D}$ by computing the samples of the fields produced by mirrors of the chip on the sampling patch. We made extensive use of the formulas developed in sec. 3.1 for that purpose. In our case, size of $\mathbf{D}$ became $625 \times 15123$. (There are $71 \times 71 \times 3=15123$ different fields and we took $25 \times 25=625$ samples for each field.) Vector $b(625 \times 1)$ contains the samples of target field. We tried to solve $\mathbf{p}$.

The reconstruction we obtained is shown in Fig. 3. The final error is $5.8 \%$.

While still there are many open questions waiting to be answered about proper application of the developed framework, this initial simulation suggests that our approach is handy in dealing with this synthesis problem.

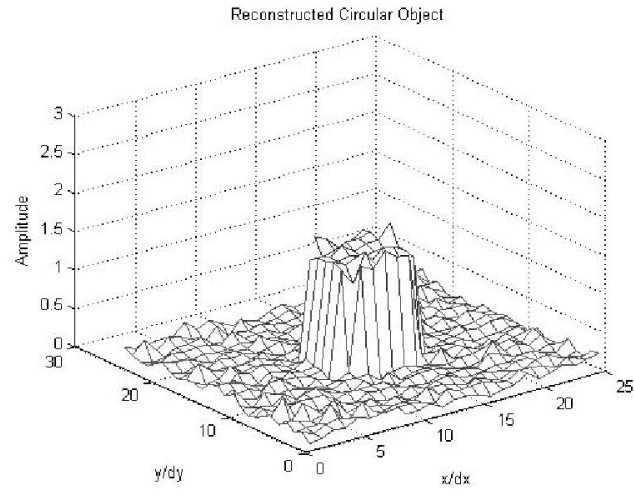

Fig. 3. Reconstructed field

\section{CONCLUSION}

This work contains efforts to solve the two central problems associated with holographic 3DTV. The proposed algorithm for the first problem can be used to calculate diffraction pattern over $2 \mathrm{D}$ space from an arbitrary curve. The algorithm provides linear solution and its computational complexity is dominated by the computation of the pseudo-inverse of $B_{B F}$ matrix. For the second part, we undertook the light field synthesis problem with a DMAD. An analysis of the light field generated by the device is given. The derived formulas are useful in calculating the field of the mirrors to be utilized during the solution of the inverse problem. At a first glance, the inverse problem seems to be complex. However, we have shown that it can be cast in the format of a linear constrained optimization problem, for which many fast algorithms exist and can be adapted.

\section{REFERENCES}

[1] M. L. Huebschman, B. Munjuluri, and H. R. Garner, "Dynamic holographic 3-d image projection," Optics Express, vol. 11, pp. 437-445, 2003.

[2] J. W. Goodman, Introduction to Fourier Optics, McGraw-Hill, New York, 1996.

[3] L. Onural and P. D. Scott, "Digital decoding of in-line holograms," Optical Engineering, vol. 26, pp. 11241132, 1987.

[4] L. J. Hornbeck, "Digital light processing update: status and future applications," in Conf on Projection Displays V, Proc SPIE, 1999, vol. 3634, pp. 158-170.

[5] L. Onural, "Sampling of the diffraction field," Applied Optics, vol. 39, pp. 5929-5935, 2000. 